\title{
Facet Reflectivity of a Spot-Size-Converter Integrated Semiconductor Optical Amplifier
}

\author{
Jongin Shim, Member, IEEE, Jongryeol Kim, Donghoon Jang, Yungseon Eo, Member, IEEE, and \\ Shigehisa Arai, Member, IEEE
}

\begin{abstract}
Traveling-wave type semiconductor optical amplifiers (SOAs) integrated with a spot-size-converter (SSC) are extensively studied for the improvement of coupling efficiency with single-mode fiber and for cost reduction in packaging. In this paper, the structural dependence of the SSC on the effective facet reflectivity $R_{\text {eff }}$ is investigated theoretically as well as experimentally. It is shown that not only a sufficient mode-conversion in a SSC region, but also an introduction of angled facets, are essential for reducing $R_{\text {eff }}$. A small gain ripple (less than $0.1 \mathrm{~dB}$ ) in an amplified spontaneous emission (ASE) spectrum, fiber-to-fiber gain of $26 \mathrm{~dB}$, and saturation output power of $7 \mathrm{dBm}$ are observed at the fabricated SOA, which consists of a window length of $20 \mu \mathrm{m}$, facet angle of $7^{\circ}$, and anti-reflection coated facet of less than $1 \%$ reflectivity.
\end{abstract}

Index Terms-Angled facet, facet reflectivity, semiconductor optical amplifier, spot-size-converter, window.

\section{INTRODUCTION}

$\mathbf{S}$ EMICONDUCTOR optical amplifiers (SOAs) are considered attractive components for future optical communication systems, since they are able to perform various functions ranging from linear amplification to all-optical processing [1]-[3]. SOAs are classified into two groups: Fabry-Perot amplifiers (FPAs) and traveling-wave amplifiers (TWAs). The major difference between the two is the facet reflectivities of the end facets. The TWAs are preferred over the FPAs due to their superior characteristics in bandwidth, saturation output power, and noise figure. The reduction in facet reflectivity is a key step in the development of high-performance TWAs. Residual facet reflectivity can appear in a number of problems, including gain ripple and saturation, excess noise, and increased polarization sensitivity [4], [5].

A single-layer anti-reflection (AR) coating requires a quarter-wave film with a refractive index equal to the square root of the refractive index of the substrate, i.e., $n_{f}=\sqrt{n_{s}}$ and $h_{f}=\lambda / 4 n_{f}$. Here, $n_{f}$ and $n_{s}$ are the refractive indices of the AR-coated film and the substrate, respectively, $h_{f}$ is the

Manuscript received October 26, 2001; revised March 11, 2002. This work was supported in part by the Korea Science and Engineering Foundation (KOSEF) through the Center for Electronic Packaging Material (CEPM), KAIST, Korea.

J. Shim and Y. Eo are with the Department of Electrical and Computer Engineering, Hanyang University, Ansan-Si, Kyungki-Do, Korea (e-mail: jishim@giga.hanyang.ac.kr).

J. Kim and D. Jang are with the Optoelectronics Division, Samsung Electronics Company, Ltd., Kyungki-Do, Korea.

S. Arai is with the Research Center for Quantum Effect Electronics, Tokyo Institute of Technology, Meguro-Ku, Tokyo, Japan

Publisher Item Identifier S 0018-9197(02)05032-7. thickness of the AR coated film, and $\lambda$ is the wavelength of the light in vacuum. Although a facet reflectivity of approximately $10^{-3}$ can be obtained only by using a single-layer dielectric film, the use of multilayer coatings seems to be inevitable, since the bandwidth of the single-layer is inherently very narrow [6]. Furthermore, AR coating conditions of the guided wave in a TWA are different from those for the simple plane wave since the guided mode is composed of elementary plane waves with different wave vectors. This makes it difficult to realize low facet reflectivity reproducibly and, thus, requires a complex in-situ monitoring procedure of the refractive index and film thickness. To easily obtain sufficiently low modal facet reflectivity for a wide wavelength range, various techniques have been reported, such as tilting the waveguide [7], introducing the window region [8], and combining AR coating with others [9]-[13].

The cost of the TWA is currently very high due to high precision active alignment and complicated AR coatings on the facets. The high precision active alignment is required since the coupling loss between chips and single-mode fiber is large. Also, the alignment tolerance is as small as less than $\pm 1 \mu \mathrm{m}$, which results in a high packaging cost in an SOA module. This is mainly due to the mode-size difference between the SOA and the single-mode fiber (SMF). The guided optical mode sizes from the chip and SMF are about 1 and $8 \sim 10 \mu \mathrm{m}$, respectively. The integration of a spot-size converter (SSC) with an SOA is essential for low-cost packaging issues to achieve both high coupling efficiency as well as high device yield. Thus, recently the TWA integrated with SSC has been extensively studied. In addition, the use of integrated mode expanders has potential to reduce the effective facet reflectivity when used in conjunction with window structures and angled facets [12], [13]. However, the dependence of the facet reflectivity on the structural parameters in a TWA integrated with SSC has not been clarified yet.

This paper reports the effective facet reflectivity in a TWA integrated with a SSC, and is especially focused on the facet structures and high-performances of the optimized devices. This paper is organized as follows. In Section II, the device structure and analysis method of the facet reflectivity are presented. The facet structure consists of the SSC region, window region, angled facet, and AR-coated dielectric films. The 3-D nature of the optical waveguide is taken into account. The facet reflectivity is calculated by the method based on plane-wave decomposition of the guided mode and Fresnel reflection at the discontinuities along the longitudinal axis. In Section III, the analysis and experimental results including gain characteristics are presented. Section IV gives the conclusions. 


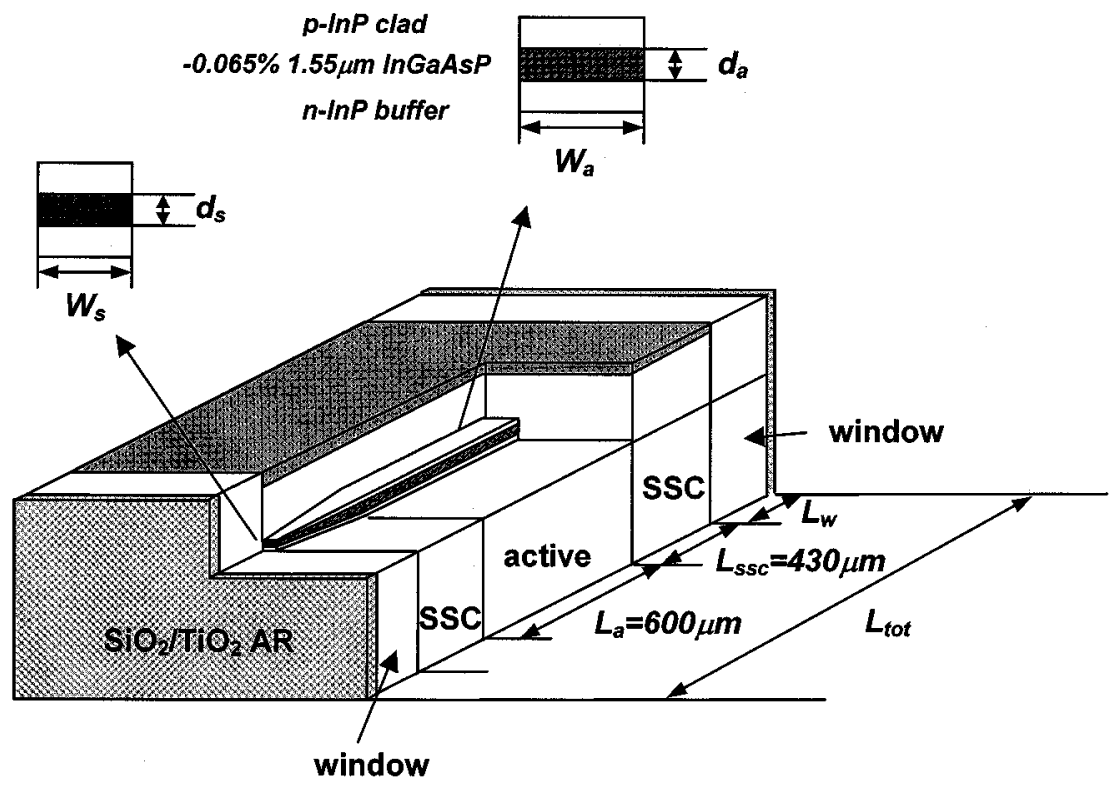

Fig. 1. Schematic of the SSC integrated SOA analyzed and fabricated.

\section{ANALYSIS OF FACET REFLECTIVITY}

\section{A. Analysis Model}

Fig. 1 shows the schematic structure of a $1.55-\mu \mathrm{m}$ SSC-SOA used in the theoretical and experimental analysis. The device consists of three regions, namely the active region, SSC region, window region, and AR-coated films. A tensile strained bulk $\operatorname{InGaAsP}\left(\lambda_{g}=1.55 \mu \mathrm{m}\right)$ layer is used as an active layer in both the straight and tapered regions. The mode expander consists of la inearly tapered active region followed by an InP window region of length $L_{w}$. Wet chemical etching is used to form the linearly tapered region. The waveguide has an angle $\theta$ with respect to the cleaved facets. The facet is AR coated using dielectric films. It has a reflectivity $R_{f}$ at the boundary between the InP window region and the dielectric films for $1.55-\mu \mathrm{m}$ wavelength light.

\section{B. Effective Facet Reflectivity $R_{\mathrm{eff}}$}

The dependence of the effective facet reflectivity $R_{\text {eff }}$ at the end of the SSC region on the facet structures is analyzed in detail. Fig. 2 shows the analysis model. Our analysis approach for calculating the modal reflectivity at the interface between the different regions is based on the plane-wave decomposition of the dominant waveguide mode and the application of Fresnel's law. If the guided mode and reflected fields have the same propagation constant $\beta$ in the $-z$ direction, the modal reflectance $R_{\mathrm{eff}}$ of the facet structure at the interface $z=0$ can be obtained from the overlap integral of the guided-mode field and the reflected field profiles as

$$
\begin{aligned}
& R_{\mathrm{eff}}= \\
& \int_{-\infty}^{\infty} \int_{-\infty}^{\infty}\left|\vec{E}_{i}(x, y)\right|^{2} d x d y \int_{-\infty}^{\infty} \int_{-\infty}^{\infty}\left|\vec{E}_{r}(x, y)\right|^{2} d x d y
\end{aligned}
$$

where $\vec{E}_{i}(x, y) e^{-j \beta z}$ and $\vec{E}_{r}(x, y) e^{j \beta z}$ are the incident and reflected electric fields at $z=0$, respectively [14]. Three major

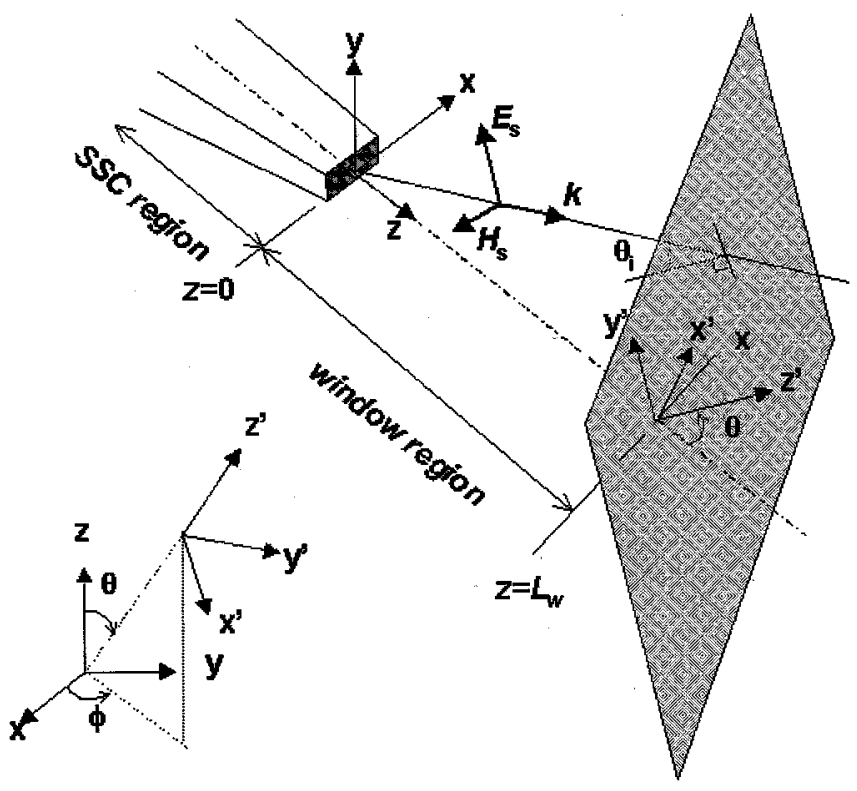

Fig. 2. Analytic model of effective reflectivity $R_{\text {cff }}$ in SSC-SOA.

approximations are used in the following analysis in order to simplify the analysis.

1) $\vec{E}_{i}(x, y)$ is assumed as the fundamental guided mode at the end of the SSC region (i.e., $z=0_{-}$). This assumption is valid if the waveguide structure of the SSC region should change adiabatically for the field to be converted into a stable fundamental mode. The fundamental quasi-TE mode is considered to be the guided mode of SSC region. The choice of quasi-TE and quasi-TM modes depends on the input light polarization. The analysis procedure and results are not so different between the modes.

2) The multiple reflection effect in the window region is neglected. In our model (as shown in Fig. 1) the facet structure consists of the SSC region, window region, and angled-facet region. As the number of reflections increases within the window region, the center of the reflected field 
will be far apart from the SSC waveguide center at $z=$ 0 by the angled-facet and window-region effects. Furthermore, the reflectivity of the field propagating to the SSC region at $z=0$ is very small, since there exists little difference between the refractive indices for the SSC and window regions. Thus, a negligible amount of field components will be coupled to the guided mode of the SSC region by the multiple reflections. Thus, we assume that the initial reflected beam at the angled facet affects $\vec{E}_{r}(x, y) e^{j \beta z}$.

3) The refractive index of the SSC region is assumed to be uniform for the transmitted field from the window region to the SSC region. Under these approximations, the total reflected field $\vec{E}_{r}(x, y) e^{j \beta z}$ is expressed as

$$
\vec{E}_{r}(x, y) e^{j \beta z}=\left\{\vec{E}_{r 1}(x, y)+\vec{E}_{r 2}(x, y)\right\} e^{j \beta z}
$$

where $\vec{E}_{r 1}(x, y) e^{j \beta z}$ and $\vec{E}_{r 2}(x, y) e^{j \beta z}$ represent the reflected field component of the incident beam $\vec{E}_{i}(x, y) e^{-j \beta z}$ at $z=0$ and $z=L_{w}$, respectively.

\section{Incident Field Profile $\vec{E}_{i}(x, y, z=0)$}

The fundamental guided mode of the SSC region at $z=$ $0_{-}$is assumed to be the quasi-TE mode. The 2-D optical field profile at $z=0_{-}$is obtained by the weighted-index method (WIM) [15], [16]. The electric field $\vec{E}(x, y, z)$ and magnetic field $\vec{H}(x, y, z)$ propagating along the $+z$ direction are expressed as

$$
\begin{aligned}
\vec{E}(x, y, z) & =\left\{E_{x}(x, y) \vec{x}+E_{z}(x, y) \vec{y}\right\} e^{-j \beta z} \\
\vec{H}(x, y, z) & =\left\{H_{x}(x, y) \vec{x}+H_{y}(x, y) \vec{y}+H_{z}(x, y) \vec{z}\right\} e^{-j \beta z}
\end{aligned}
$$

where the dominant components are $E_{x}(x, y)$ and $H_{y}(x, y)$. The electric field profile $E_{x}(x, y)$ and propagation constant $\beta$ can be obtained as a solution of the Helmholtz equation as

$$
\frac{\partial^{2} E_{x}(x, y)}{\partial x^{2}}+\frac{\partial^{2} E_{x}(x, y)}{\partial y^{2}}=\left\{\beta^{2}-k_{o}^{2} n^{2}(x, y)\right\} E_{x}(x, y)
$$

where $n(x, y)$ denotes the refractive index profile and $k_{o}$ is the free-space propagation constant. The solution using WIM is assumed to be of the form $E_{x}(x, y)=F(x) G(y)$, where $\int_{-\infty}^{\infty} F^{2} d x=\int_{-\infty}^{\infty} G^{2} d y=1$. The separation of variables makes it possible for the solution to be expressed analytically.

\section{Reflection and Transmission at the Hetero-Interface}

Since the guiding of the waveguide is bound, the plane-wave reflection coefficient cannot be directly used. However, the mode can be Fourier-transformed into an infinite sum of plane waves with the transverse wavevector $k_{x}$ and $k_{y}$. Thus, the wavevector $\vec{k}$ of each plane wave can be considered as $\left(k_{x}, k_{y}, \beta\right)$. Each plane wave has its own effective index of refraction $n_{\mathrm{eff}}(\vec{k})$, where $k_{x}^{2}+k_{y}^{2}+\beta^{2}=n_{\text {eff }}^{2}(\vec{k}) k_{o}^{2}, k_{o}=2 \pi / \lambda$, and $\lambda$ is the wavelength in space. However, the method using a different value of $n_{\text {eff }}(\vec{k})$ makes it difficult to apply Fresnel's law at the hetero-interface for forward and backward propagating waves, simultaneously [17]-[19]. Some previous studies had simplified by approximating the refractive index inside the waveguide region as a constant of $n_{\mathrm{eff}}(\vec{k}=0)=\beta / k_{o}$ [20]. This is a good approximation in the limit for a weakly guide mode, namely $\left|k_{x}\right| \ll \beta$ and $\left|k_{y}\right| \ll \beta$. A more exact approach of "zero thickness fictitious layer" has been presented in [21]. The unperturbed optical waveguide mode propagates into the infinitesimally thin fictitious layer with a constant refractive index $\bar{n}$. An optimum $\bar{n}$ is chosen in order for the residual reflection at the epitaxial/fictitious layer interface to be negligible. In our analysis, the following assumptions are made in order to find a reflectivity at the interface $z=0$ for forward and backward propagating waves. For plane waves propagating from the SSC region to the window region, a plane wave in the SSC region is assumed to carry its own refractive index $n_{\mathrm{eff}}(\vec{k})$. However, for plane waves propagating in the reverse direction, the refractive index of the SSC region is assumed to be a constant value $n_{\text {eff }}(\vec{k}=0)$. This approximation makes it possible to use Fresnel's law for both the forward and backward propagation waves.

Next, we will describe the detailed procedure used in our analysis. The angular spectrum function of the input optical field into the window region is given by the Fourier transform of $E_{x}(x, y)$ as

$$
E_{x k}\left(k_{x}, k_{y}\right)=\left(\frac{1}{2 \pi}\right)^{2} \int_{-\infty}^{\infty} \int_{-\infty}^{\infty} d x d y E_{x}(x, y) e^{j\left(k_{x} x+k_{y} y\right)}
$$

where $k_{x}^{2}+k_{y}^{2}+\beta^{2}=k_{o}^{2} n_{\text {eff }}^{2}\left(k_{x}, k_{y}, \beta\right)$ and $E_{x}(x, y)$ is the solution of (5). Thus, $E_{x k}\left(k_{x}, k_{y}\right) e^{-j\left(k_{x} x+k_{y} y+\beta z\right)}$ is considered to be a plane wave traveling in a uniform medium of $n_{\text {eff }}(\vec{k})$, where $\vec{k}=\left(k_{x}, k_{y}, \beta\right)$. Other electromagnetic field components with a wavevector $\vec{k}$ should satisfy the following Maxwell's equations that determine the quasi-TE mode field profiles, namely

$$
\begin{aligned}
E_{y k}\left(k_{x}, k_{y}\right) & \equiv 0 \\
E_{z k}\left(k_{x}, k_{y}\right) & =-\frac{k_{x}}{\beta} E_{x k} \\
H_{x k}\left(k_{x}, k_{y}\right) & =-\frac{k_{x} k_{y}}{\omega \mu_{o} \beta} E_{x k} \\
H_{y k}\left(k_{x}, k_{y}\right) & =\frac{k_{x}^{2}+\beta^{2}}{\omega \mu_{o} \beta} E_{x k} \\
H_{z k}\left(k_{x}, k_{y}\right) & =-\frac{k_{y}}{\omega \mu_{o}} E_{x k}
\end{aligned}
$$

where $\omega$ and $\mu_{o}$ are the angular frequency and the permeability in vacuum, respectively.

Thus, a plane wave with $\vec{k}$ can be expressed as

$$
\begin{aligned}
\vec{E}_{k} & =\left\{E_{k x} \vec{x}+E_{k z} \vec{z}\right\} e^{-j\left(k_{x} x+k_{y} y+\beta z\right)} \\
\vec{H}_{k} & =\left\{H_{k x} \vec{x}+H_{k y} \vec{y}+H_{k z} \vec{z}\right\} e^{-j\left(k_{x} x+k_{y} y+\beta z\right)}
\end{aligned}
$$

where $\vec{E}_{k}, \vec{H}_{k}$, and $\vec{k}$ are orthogonal to each other.

In order to apply Frensel's law easily at the interface (i.e., $z=0$ ), it is necessary to decompose $\vec{E}_{k}$ and $\vec{H}_{k}$ into $s$ and $p$ waves. The resulting expressions of $\vec{E}_{k}$ and $\vec{H}_{k}$ are

$$
\begin{aligned}
& \vec{E}_{k}=\left(\vec{E}_{k} \bullet \vec{e}_{s}\right) \vec{e}_{s}+\left(\vec{E}_{k} \bullet \vec{e}_{p}\right) \vec{e}_{p}=E_{s} \vec{e}_{s}+E_{p} \vec{e}_{p} \\
& \vec{H}_{k}=\left(\vec{H}_{k} \bullet \vec{h}_{s}\right) \vec{h}_{s}+\left(\vec{H}_{k} \bullet \vec{h}_{p}\right) \vec{h}_{p}=H_{s} \vec{h}_{s}+H_{p} \vec{h}_{p}
\end{aligned}
$$


where $\vec{e}_{s}, \vec{e}_{p}, \vec{h}_{s}$, and $\vec{h}_{p}$ are unit vectors. Both $\vec{e}_{s}$ and $\vec{h}_{p}$ lie on the $x-y$ plane as shown in Fig. 2. The unit vectors and amplitudes of $s$ and $p$ waves are related to each other as follows:

$$
\begin{aligned}
\vec{e}_{s} \times \vec{h}_{s} & =\vec{k}_{n} \\
\vec{e}_{p} \times \vec{h}_{p} & =\vec{k}_{n} \\
\frac{H_{s}}{E_{s}}=\frac{H_{p}}{E_{p}} & =\frac{1}{\eta}
\end{aligned}
$$

where $\vec{k}_{n}=\vec{k} /|\vec{k}|, \eta=\left(1 / n_{\mathrm{eff}}(\vec{k})\right) \sqrt{\mu_{o} / \varepsilon_{o}}$, and $\varepsilon_{o}$ is the permittivity in vacuum. It is noted that the choice of $\vec{e}_{s}$ and $\vec{h}_{p}$ is not unique. Here, $\vec{e}_{s}$ and $\vec{h}_{p}$ are defined by the projection of $\vec{E}_{k}$ and $\vec{H}_{k}$ onto the $x-y$ plane. Therefore

$$
\begin{aligned}
& \vec{e}_{s}=\vec{x} \\
& \vec{h}_{p}=\frac{H_{k x}}{\sqrt{H_{k x}^{2}+H_{k y}^{2}}} \vec{x}+\frac{H_{k y}}{\sqrt{H_{k x}^{2}+H_{k y}^{2}}} \vec{y} .
\end{aligned}
$$

Fresnel's law finds the reflection and transmission coefficients for the $s$ and $p$ waves at the interface $z=0$ to be

$$
\begin{aligned}
r_{s} & =\frac{n_{\mathrm{eff}} \cos \theta_{i}-\sqrt{n_{2}^{2}-n_{1}^{2} \sin ^{2} \theta_{i}}}{n_{\mathrm{eff}} \cos \theta_{i}+\sqrt{n_{2}^{2}-n_{1}^{2} \sin ^{2} \theta_{i}}} \\
r_{p} & =-\frac{n_{w} \cos \theta_{i}-\left(\frac{n_{1}}{n_{2}}\right) \sqrt{n_{2}^{2}-n_{1}^{2} \sin ^{2} \theta_{i}}}{n_{\mathrm{eff}} \cos \theta_{i}+\left(\frac{n_{1}}{n_{2}}\right) \sqrt{n_{2}^{2}-n_{1}^{2} \sin ^{2} \theta_{i}}} \\
t_{s} & =1+r_{s} \\
t_{p} & =\frac{\cos \theta_{i}}{\cos \theta_{t}}\left(1+r_{s}\right) .
\end{aligned}
$$

The incident angle $\theta_{i}$ and transmitted angle $\theta_{t}$ are given by

$$
\begin{aligned}
\cos \theta_{i} & =\frac{\sqrt{k_{x}^{2}+k_{y}^{2}}}{\sqrt{k_{x}^{2}+k_{y}^{2}+k_{z}^{2}}} \\
\cos \theta_{t} & =\sqrt{1-\frac{n_{n e f f}^{2}}{n_{w}^{2}} \sin ^{2} \theta_{i} .}
\end{aligned}
$$

\section{E. Propagation in the Window Region}

There exist plane waves propagating in the $\pm z$ directions in the window region. In our model, plane waves propagating in the $+z$ direction are transmitted fields through the plane at $z=0$ from the SSC region to the window region. In addition, plane waves propagating in the $-z$ direction result in the reflected fields at the angle facet. Let $\vec{E}_{1}\left(x, y, z=0_{+}\right)$and $\vec{E}_{1 k}\left(k_{x}, k_{y}\right)$ be the transmitted electric field at $z=0$ of $\vec{E}_{i}\left(x, y, z=0_{-}\right)$ of the incident field interface and its angular spectrum vector, respectively. Then the electric field distribution after traveling from $z=0$ to $z=L_{w}$ can be written as

$$
\begin{aligned}
\vec{E}_{1} & \left(x, y, z=L_{w}\right) \\
= & \int_{-\infty}^{\infty} \int_{-\infty}^{\infty} d k_{x} d k_{y} \vec{E}_{1 k}\left(k_{x}, k_{y}\right) \\
& \cdot \exp \left\{-j\left(k_{x} x+k_{y} y+\sqrt{\left(k_{o} n_{w}\right)^{2}-k_{x}^{2}-k_{y}^{2}} L_{w}\right)\right\}
\end{aligned}
$$

where $n_{w}$ is the refractive index in the window region. The wave set in (27) consists of plane waves $\left(k_{o}^{2} n_{w}^{2} \geq k_{x}^{2}+k_{y}^{2}\right)$ and evanescent waves $\left(k_{o}^{2} n_{w}^{2}<k_{x}^{2}+k_{y}^{2}\right)$. The evanescent waves decay exponentially along the $+z$ direction. In order to calculate the reflection from the angled facet and air interface, the coordinate transformation from an unprimed to a primed coordinate system is required. For a plane wave exiting the angled facet, it will pass through the angled facet if $k_{o} \geq \sqrt{k_{x}^{\prime 2}+k_{y}^{\prime 2}}$. It will be totally reflected at the angled-facet interface if $k_{o}<$ $\sqrt{k_{x}^{\prime 2}+k_{y}^{\prime 2}}$. When the interface is tilted at angles $\theta$ and $\phi$ as shown in Fig. 2, $\vec{E}(x, y, z), \vec{H}(x, y, z)$, and $\vec{k}$ can be transformed into $\vec{E}^{\prime}\left(x^{\prime}, y^{\prime}, z^{\prime}\right), \vec{H}^{\prime}\left(x^{\prime}, y^{\prime}, z^{\prime}\right)$, and $\vec{k}^{\prime}$, respectively [22]. In a primed coordinate system

$$
\begin{aligned}
& \vec{E}^{\prime}\left(x^{\prime}, y^{\prime}, z^{\prime}\right) \\
& \quad=\left\{E_{x}^{\prime}\left(x^{\prime}, y^{\prime}\right) \vec{x}^{\prime}+E_{y}^{\prime}\left(x^{\prime}, y^{\prime}\right) \vec{y}^{\prime}+E_{z}^{\prime}\left(x^{\prime}, y^{\prime}\right) \vec{z}\right\} e^{-j k_{z}^{\prime} z^{\prime}} \\
& \quad \vec{H}^{\prime}\left(x^{\prime}, y^{\prime}, z^{\prime}\right) \\
& \quad=\left\{H_{x}^{\prime}\left(x^{\prime}, y^{\prime}\right) \vec{x}^{\prime}+H_{y}^{\prime}\left(x^{\prime}, y^{\prime}\right) \vec{y}^{\prime}+H_{z}^{\prime}\left(x^{\prime}, y^{\prime}\right) \vec{z}\right\} e^{-j k_{z}^{\prime} z^{\prime}} .
\end{aligned}
$$

The transformation between primed and unprimed coordinate systems is given by

$$
\left[\begin{array}{l}
x^{\prime} \\
y^{\prime} \\
z^{\prime}
\end{array}\right]=\left[\begin{array}{ccc}
\cos \theta \cos \phi & \cos \theta \sin \phi & -\sin \theta \\
-\sin \phi & \cos \phi & 0 \\
\sin \theta \cos \phi & \sin \theta \cos \phi & \cos \theta
\end{array}\right]\left[\begin{array}{l}
x \\
y \\
z
\end{array}\right] .
$$

The effective facet reflectivity $R_{\text {eff }}$ in (1) is determined by the larger value between two reflected field components from the interfaces $z=0$ and $z=L_{w}$, i.e., $E_{r 1}(x, y, 0)$ and $E_{r 2}(x, y, 0)$, respectively. The dependence of $E_{r 1}(x, y, 0)$ and $E_{r 2}(x, y, 0)$ on the facet structure is presented in the following section.

\section{RESULTS AND DISCUSSIONS}

The facet structure of SOA modeled in this paper consists of a SSC region, window region, and angled facet as shown in Fig. 1. When the light is emitted from the SSC region, reflections occur at the two interfaces of $z=0$ and $z=L_{w}$. The reflection at the interface between the SSC and window regions (i.e., $z=0$ ) is mainly determined by the equivalent refractive index difference between two regions. Fig. 3 shows the calculated facet reflectivity $R_{1}$, which comes from the reflected field component at $z=0$, i.e., $E_{r, 1}$. In this analysis, the interface plane at $z=0$ is assumed to be perpendicular to the $z$ axis, namely $\theta=\phi=0^{\circ}$ and the refractive index of an InP window region to be 3.17 for $1.55-\mu \mathrm{m}$ light. As the waveguide width $W_{s}$ or thickness $d$ decreases, $R_{1}$ and $\Gamma$ drastically decrease with expanding mode size. It is known that $R_{\mathrm{eff}}<5 \times 10^{-5}$ is necessary in order to suppress gain ripple sufficiently in traveling-wave type semiconductor amplifiers [5], [23]. Thus, the values of $W_{s}$ and $d$ should be chosen such that $R_{1} \leq 5 \times 10^{-5}$. For example, $W_{s} \leq 0.3 \mu \mathrm{m}$ and $d=0.2 \mu \mathrm{m}$ can be a good choice since a laterally tapered SSC structure can be easily realized only by conventional photolithography and wet etching techniques. Fig. 4 shows the optical confinement factor $\Gamma$ of various waveguide structures at $z=0_{-}$. The straight lines are also plotted to indicate the condition $R_{1} \leq 10^{-5}$ and $R_{1} \leq 10^{-4}$. A weakly 


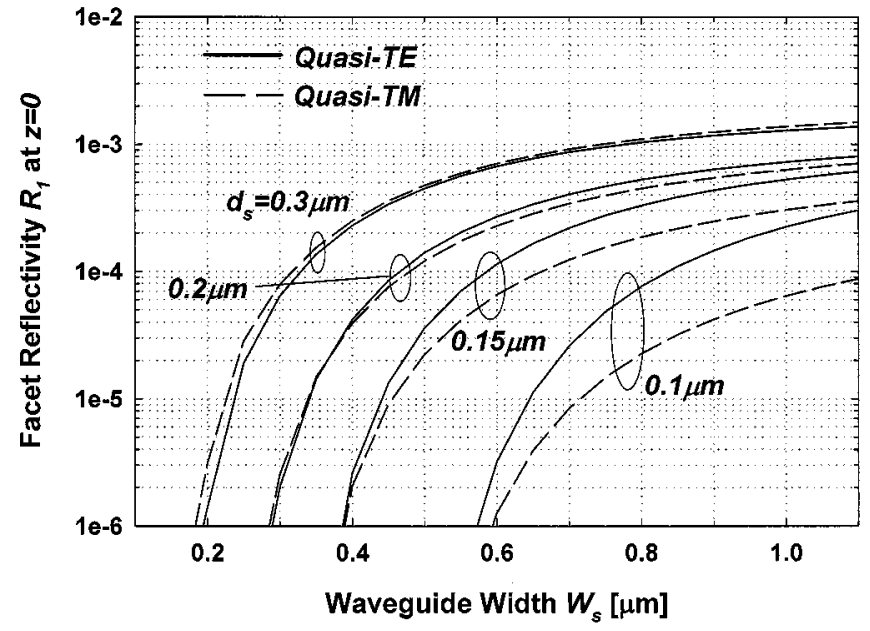

Fig. 3. Calculated facet reflectivity $R_{1}$ at $z=0$ according to the waveguide width $W_{s}$.

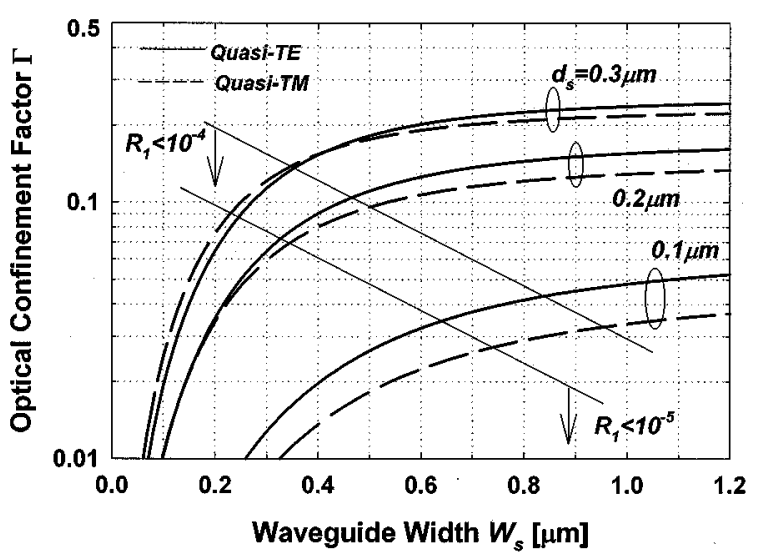

Fig. 4. Calculated optical confinement factor $\Gamma$ according to the waveguide width $W_{s}$.

guided SSC $(\Gamma \leq 0.1)$ needs to be integrated with the window structure both to prevent from the reflection at $z=0$ and to achieve high optical coupling with a single-mode fiber.

Another field component to influence the facet reflectivity is the light reflected at the angled facet of $z=L_{w}$, i.e., $\vec{E}_{r 2}$. The magnitude of $\vec{E}_{r 2}$ depends on the window and angle-facet structures. Commonly, dielectric films needs to be coated at the angle facet in order to obtain extremely low reflectivity. The plane wave reflectivity owing to coated dielectric films varies with the wavevector $\vec{k}$. Therefore, the facet reflectivity of each plane wave by dielectric films needs to be considered separately for an exact analysis. A popular method used is the transfer matrix method [6], [18]. In our analysis, we neglect the detailed effect of dielectric film structure in order to concentrate on the window structure and facet tilting effects. The dielectric film region is assumed to be uniform medium with a fictitious refractive index $n_{f}$ of 2.56. This value gives a power reflectivity $R_{f}$ of about $1.0 \%$ for a normal incident plane wave from the window region to the dielectric film region. The value $R_{f} \leq 1 \%$ can be realized simply by a single-pair dielectric coating. Fig. 5 shows the facet reflectivity $R_{2}$ at $z=0$ according to the window region length $L_{w}$. The values $W_{s}=0.3 \mu \mathrm{m}$ and $\theta=\phi=0^{\circ}$ are used in this

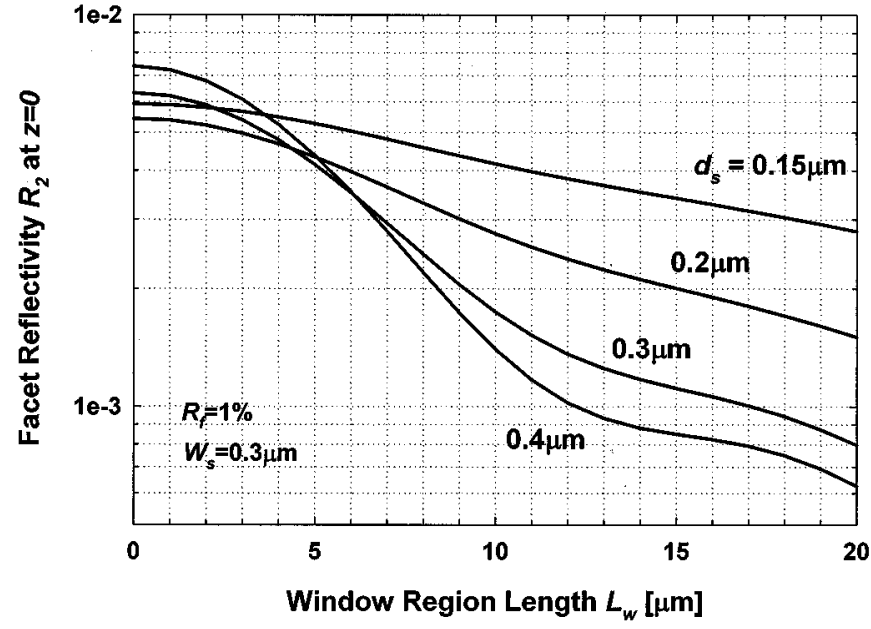

Fig. 5. Calculated facet reflectivity $R_{2}$ at $z=0$ according to the window region length $L_{w}$.

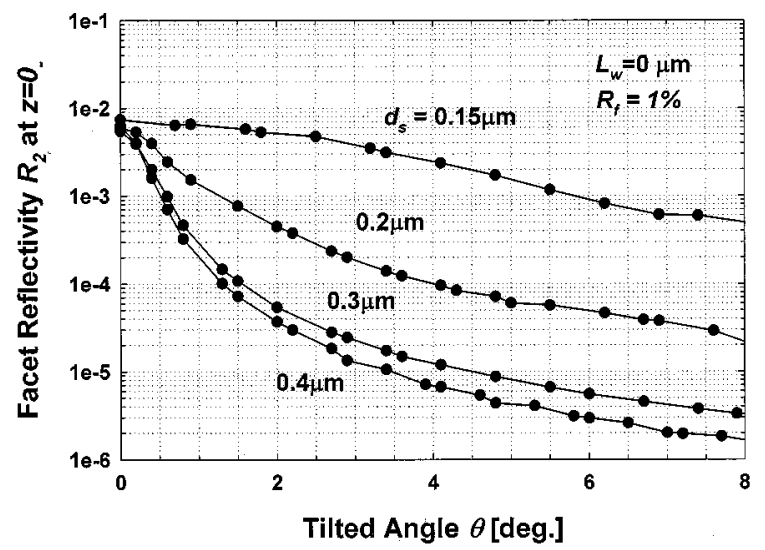

Fig. 6. Calculated facet reflectivity $R_{2}$ at $z=0$ according to the tilted angle $\theta$ without window region.

calculation. Also, $R_{2}$ decreases monotonically as $L_{w}$ increases. Furthermore, the effect of reducing $R_{2}$ by $L_{w}$ appears stronger as waveguide thickness $d_{s}$ increases. The strongly confined optical field profile in the waveguide consists of the plane waves with large parallel wavevector $\vec{k}_{x}\left(\vec{k}_{y}\right)$ components. Therefore, the stronger the field is confined, the larger the field is dispersed during the propagation within the window region. Even though $R_{2}$ decreases as $d_{s}$ increases, the maximum permissible value of $d_{s}$ is usually limited by the optical gain in the straight active region and the facet reflectivity $R_{1}$. From Fig. 6, the maximum useful value of $d_{s}$ is about $0.2 \mu \mathrm{m}$ under the condition $R_{2} \leq 10^{-5}$. It is possible to decrease $R_{2}$ at a desired value by elongating the window region length $L_{w}$. However, the maximum length of $L_{w}$ is usually determined by the far-field pattern (FFP) of the device. For the case where $L_{w}$ is too long, the optical field emitted by the SSC region will be reflected at the top plane of the cladding layer before arriving at the window region facet. The reflection from the surface region deforms FFP greatly and results in a large coupling loss between the SOA and single-mode fiber. In order to avoid the deformation of FFP, a very thick InP window layer is inevitable. However, this requires a long growth time. For these reasons, $L_{w} \leq 20 \mu \mathrm{m}$ is desirable. 


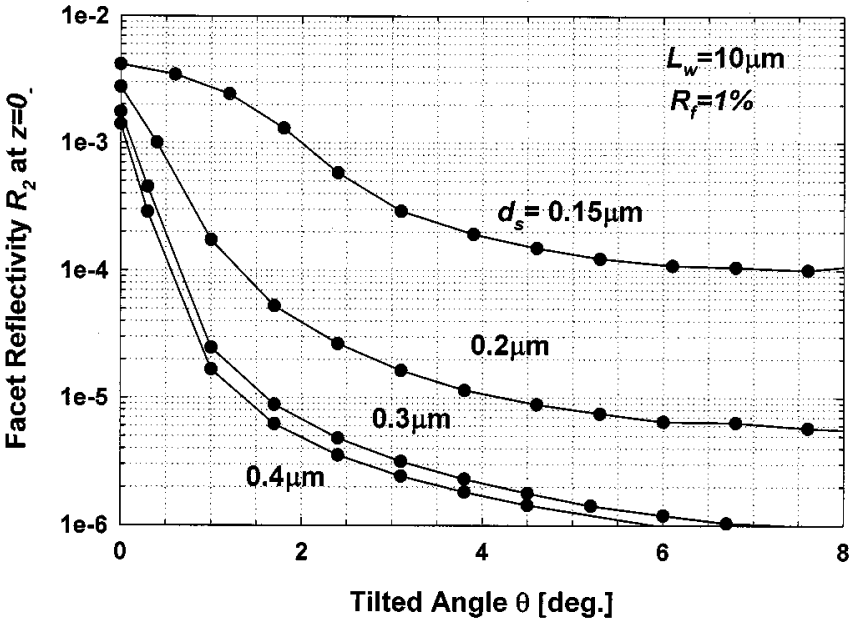

Fig. 7. Calculated facet reflectivity $R_{2}$ at $z=0$ according to the tilted angle $\theta$ with $10-\mu \mathrm{m}$ window region.

Fig. 7 shows the dependence of facet reflectivity $R_{2}$ on the facet angle $\theta$. In this analysis, the window region length $L_{w}=10 \mu \mathrm{m}$ and the facet reflectivity due to dielectric film coating having $R_{f}=1 \%$ are assumed. Therefore, the decrease of facet reflectivity results from only the angled facet. A facet reflectivity $R_{2}<10^{-5}$ can be easily obtained by introducing a thick and/or wide waveguide structure and an angled facet. The strongly confined field makes it difficult for the light to couple with a single-mode fiber due to the large and asymmetric FFP. Thus, it is better to use a weakly guiding structure around the facet region for good coupling with the fiber since it produces a more symmetric and narrower FFP compared to a strongly guiding structure. A weakly guiding structure has a disadvantage in the point of small facet reflectivity as shown in Fig. 6. Furthermore, $R_{2}$ decreases very slowly along $\theta$ in a weakly guided waveguide structure such as when $d_{s}=0.15 \mu \mathrm{m}$. Since the mode size of the weakly guided structure is very large, overlap integral between the incident field $E_{i}(x, y, z=0)$ and reflected field $E_{r, 2}(x, y, z=0)$ slightly changes by tilting the facet. In the case where $d_{s}=0.2 \mu \mathrm{m}$, the facet angling effect appears large for $\theta<4 \sim 5^{\circ}$ and slowly saturates as $\theta$ increases. Furthermore, the facet angle effect of $R_{2} / R_{f}$ is as small as $10^{-2}$, where $R_{f}$ is the reflectivity of the dielectric coating film. Therefore, it is almost impossible to realize $R_{2}<10^{-5}$ without introducing a window region or multi-layered AR films of $R_{f}<1 \%$ in a SSC with weakly waveguided structures.

Figs. 7 and 8 show the dependency of facet reflectivity $R_{2}$ on the facet angle $\theta$ for $L_{w}=10 \mu \mathrm{m}$ and $20 \mu \mathrm{m}$, respectively. The values $R_{f}=1 \%$ and $W_{s}=0.3 \mu \mathrm{m}$ are used in this calculation. It is possible to achieve $R_{2}<10^{-5}$ for $d_{s} \geq 0.2 \mu \mathrm{m}$ by adjusting the facet angle $\theta$. There exists a maximum useful value of $\theta$ for practical applications although $R_{2}$ decreases by increasing $\theta$. For example, a light beam from the angled facet of $7^{\circ}$ emits in the direction tilted by $\theta=21^{\circ}$ from the longitudinal axis. When $\theta$ is greater than $20^{\circ}$, the layout between the device and fiber is not so simple in the small packaging. Also, the optical coupling loss increases. Furthermore, $R_{2}$ decreases drastically in the range of $0^{\circ} \leq \theta \leq 4^{\circ}$. Thus, $\theta \leq 7^{\circ}$ is promising in a SSC integrated SOA. By comparing Fig. 6 with Figs. 7 and

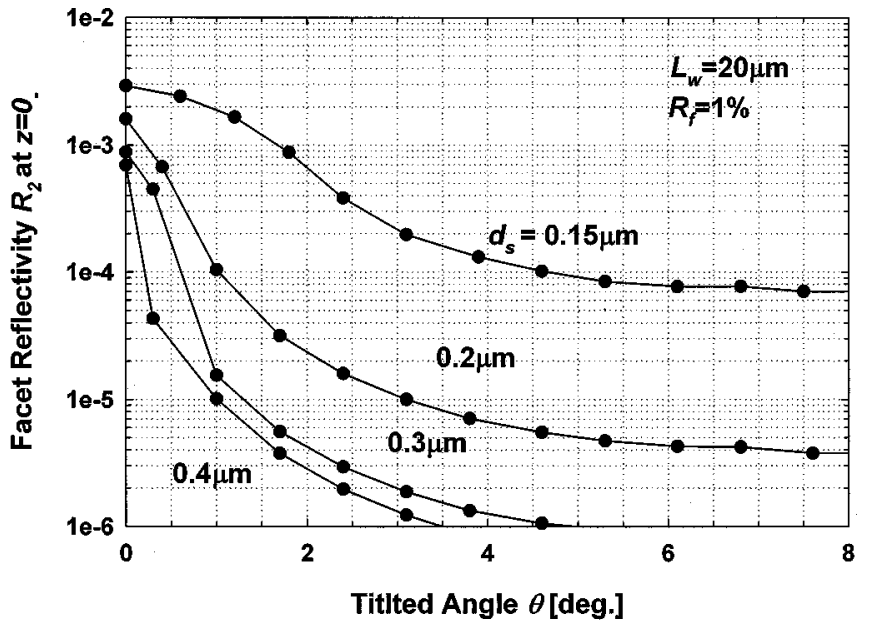

Fig. 8. Calculated facet reflectivity $R_{2}$ at $z=0$ according to the tilted angle $\theta$ with $20-\mu \mathrm{m}$ window region.

$8, R_{2} / R_{f}$ of a device with $d_{s}=0.2 \mu \mathrm{m}$ can be further reduced by a factor of $10^{-1}$ with a window region of $L_{w} \geq 10 \mu \mathrm{m}$.

A fabricated SOA integrated with a SSC, window region, and angled facet is shown in Fig. 1. The devices were grown by 3 -step low-pressure metal-organic chemical vapor deposition technologies. The total length of the device is $1.5 \mathrm{~mm}$, with an SSC at each facet and a $600-\mu \mathrm{m}$ strained active region. By taking the polarization insensitivity into account, a slightly tensile-strained $(-0.065 \%)$ and $0.2-\mu \mathrm{m}$ bulk InGaAsP $\left(\lambda_{g}=1.55 \mu \mathrm{m}\right)$ layer was used as a waveguide layer in both the straight active and the SSC regions [13]. The mode expander consists of a $430 \mu \mathrm{m}$ linearly tapered active region followed by $20 \mu \mathrm{m}$ window region. A wet chemical etching technique was used to realize the laterally tapered structures in the SSC region. The width $W_{s}$ of the active waveguide was tightly controlled to be $1.0 \mu \mathrm{m}$ in the central straight region and reduced down to about $0.2 \mu \mathrm{m}$ at the end of SSC region. The slope of the linearly tapered region was maintained below $4 \mathrm{mR}$ for an adiabatic mode size expansion. The device dimensions used in the straight active region were chosen to achieve a chip gain $G \geq 30 \mathrm{~dB}$ and the saturation output power $P_{\text {sat }} \geq 5 \mathrm{dBm}$ at the injected current $I \leq 200 \mathrm{~mA}$. In this design, we assumed that the waveguide structure of the SSC region did not change the device performances except for output beam mode size. A $5-\mu \mathrm{m}$-thick InP cladding layer is overgrown in order to be free from any reflections. The details of the waveguide dimension are summarized in Table I. The waveguide is orientated by $7^{\circ}$ to the cleaved facets. The facet is AR coated using a $\mathrm{TiO}_{2} / \mathrm{SiO}_{2}$ single-layer for all devices. The refractive indices of coated $\mathrm{TiO}_{2}$ and $\mathrm{SiO}_{2}$ films were measured by three-angle ellipsometer. The corresponding values of 2.17 and 1.46 were evaluated for a plane wave with $1.55-\mu \mathrm{m}$ wavelength. The thickness of each dielectric film was selected in order that the wavelength of minimum reflectivity for a normally incident plane wave was obtained at a wavelength slightly longer than $1.55 \mu \mathrm{m}$. This is because the wavelength showing the minimum reflectivity of spatially bounded beam occurs at shorter wavelength compared to that of normal incident plane wave. Thus, it was expected that the facet reflectivity at the boundary between the InP window 
TABLE I

Major Structural Parameters of a Fabricated SPOT-SIZE INTEGRATED SOA

\begin{tabular}{c|c}
\hline Structural Parameters & Values \\
\hline Center region length $L_{a}$ & $600 \mu \mathrm{m}$ \\
\hline SSC region length $L_{s s c}$ & $430 \mu \mathrm{m}$ \\
\hline Window region length $L_{w}$ & $20 \mu \mathrm{m}$ \\
\hline Waveguide width in the center region $W_{a}$ & $1.0 \mu \mathrm{m}$ \\
\hline Waveguide width in the end of SSC region $W_{s}$ & $\leq 0.2 \mu \mathrm{m}$ \\
\hline Waveguide thickness in the center region $d_{a}$ & $0.2 \mu \mathrm{m}$ \\
\hline Waveguide thickness in the SSC region $d_{s}$ & $\leq 0.2 \mu \mathrm{m}$ \\
\hline p-InP clad thickness $d_{c}$ & $5 \mu \mathrm{m}$ \\
\hline
\end{tabular}

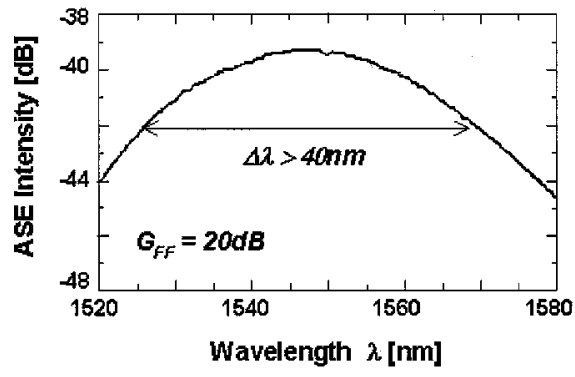

Fig. 9. ASE spectra of fabricated SOA at $100 \mathrm{~mA}$.

region and dielectric films was reduced to less than $1 \%$ by the single-layer AR coating in a fabricated device.

Fig. 9 shows the measured amplified spontaneous emission (ASE) spectrum at the current of $100 \mathrm{~mA}$. The current was injected uniformly into all active layers that existed in both a straight region and a SSC region. The resolution of the spectrum analyzer was fixed at $0.1 \mathrm{~nm}$. The peak-to-peak values of ASE ripples were measured to be as low as $0.1 \mathrm{~dB}$. This relatively low ASE ripple value means that the facet reflectivity is lower than $10^{-5}$, which also agrees with the expected results from calculations. Fig. 10 shows the measured FFPs) along the horizontal and vertical directions to the junction plane. A nearly circular FFP was observed. The full-width at half-maximum (FWHM) of the horizontal and vertical FFPs are measured as $20^{\circ} \times 20^{\circ}$. The gain measurement was performed by coupling tapered- and lensed-fibers to both facets of the device. Fig. 11 shows the fiber-to-fiber gain versus output power for the different injected current levels. A $1.55-\mu \mathrm{m}$ DFB-LD was used as an input light source. The fiber-to-fiber gain and 3-dB saturation output power were measured to be $26 \mathrm{~dB}$ and $7 \mathrm{dBm}$, respectively. The fiber-coupling loss per facet was estimated to be approximately $3 \mathrm{~dB}$. Thus, net optical gain of the fabricated SOA device was expected to be as high as $32 \mathrm{~dB}$ at $I=200 \mathrm{~mA}$.

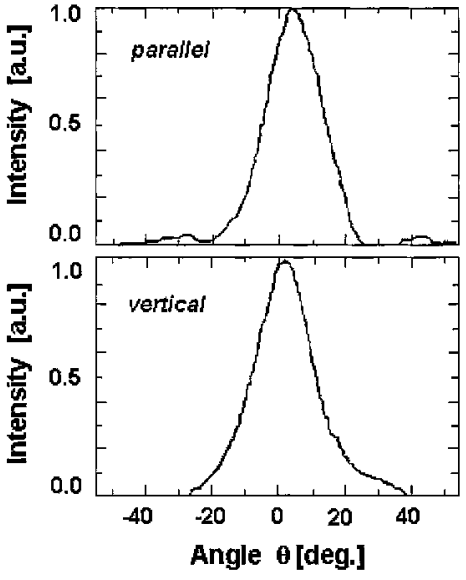

Fig. 10. FFPs of the fabricated SOA to: (a) parallel and (b) vertical directions.

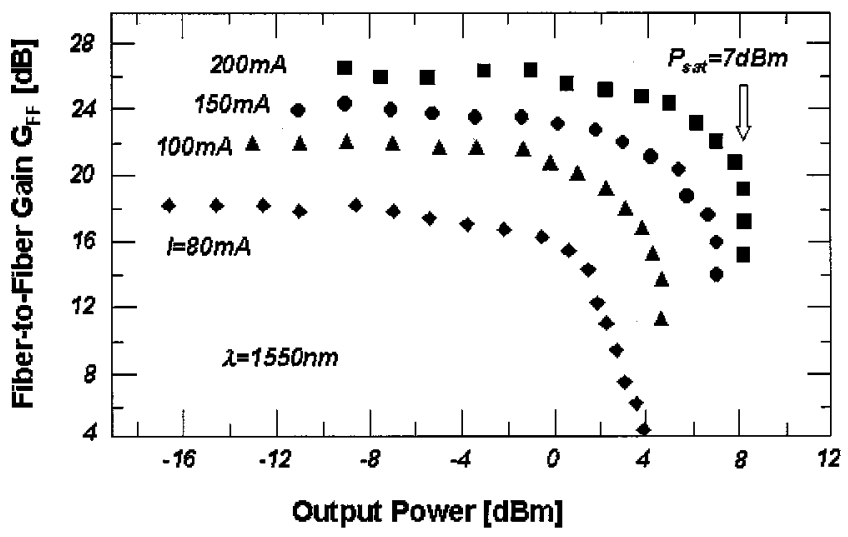

Fig. 11. Fiber-to-fiber gain of fabricated SOA at several driving currents.

These superior characteristics originated from the reduction of the facet reflectivity.

\section{CONCLUSION}

The effective facet reflectivity $R_{\text {eff }}$ of a SOA was intensively studied both theoretically and experimentally. The facet structure consisted of a SSC region, window region, AR coated film, and air region. In order to analyze the effective facet reflectivity, plane-wave decomposition techniques of the guide mode and Fresnel's Law were applied. Detailed analysis showed that the facet structure integrated with the SSC, window region, angled facet, and AR-coating was essential to achieve simultaneously high fiber-to-fiber gain with small gain ripple and high optical coupling efficiency with single-mode optical fiber. Two types of SSCs can be considered, namely strongly and weakly guiding structures. The combination of the strongly guiding SSC with a waveguide width as wide as a few micrometers and angled-facet structure was effective in reducing the facet reflectivity. However, this type of SSC usually emits asymmetric FFP, which makes it difficult to achieve high coupling efficiency with a single-mode optical fiber. Thus, a weakly guiding SSC seems to be more promising to realize high coupling efficiency with a large alignment tolerance. The optical field emitted by the weakly guiding SSC consists of almost paraxial plane waves. The spot-size of a near-field pattern is very large and FFP angles 
are very small. This property makes it difficult to achieve facet reflectivity as low as $10^{-5}$ only by the combination of anglefacet and facet reflectivity $R_{f}$ due to the AR coating. Thus, the integration of the window region, angled facet, and AR-coating spot size is needed for a weakly guiding SSC structure.

The maximum waveguide dimensions of the SSC region are chosen by the permissible reflection $R_{1}$ at the interface between the SSC region and the window region. In the model used in this paper, the waveguide width $W_{s} \leq 0.35 \mu \mathrm{m}$ and active layer thickness $d_{s} \leq 0.2 \mu \mathrm{m}$ are the conditions for $R_{1} \leq 10^{-5}$. The other reflection component comes from the interface between the InP window region and the facet. The reflectivity $R_{2}$ at this interface can be controlled by the window region length $L_{w}$, facet angle $\theta$, and the reflectivity $R_{f}$. We investigated the dependence of $R_{2}$ on $L_{w}, \theta$, and $R_{f}$. For the selected SSC structure of $W_{s}=0.3 \mu \mathrm{m}$ and $d_{s}=0.2 \mu \mathrm{m}$, the $R_{2} / R_{f}$ reduction effect is significant in the range of $L_{w} \leq 10 \mu \mathrm{m}$ and $\theta \leq 4^{\circ}$. The values of $R_{2} / R_{f}$ can be reduced by the factors of $10^{-1}$ and $10^{-2}$ with $L_{w}$ and $\theta$, respectively. Therefore, the introduction of an angled facet is considered to be more effective than a window region in reducing $R_{2}$ in a SSC integrated SOA. Finally we summarize the conditions of the facet structure for high-performance SOA as follows. The SSC waveguide width $W_{s} \leq 0.35 \mu \mathrm{m}$, SSC waveguide thickness $d_{s} \leq 0.2 \mu \mathrm{m}$, window region length $L_{w} \geq 10 \mu \mathrm{m}$, facet angle $\theta \geq 4^{\circ}$, and the reflectivity of the dielectric film $R_{f} \leq 1 \%$.

We obtained high-performances by a fabricated SOA integrated with a SSC, window region, and angled facet. The device dimensions of $W_{s} \sim 0.2 \mu \mathrm{m}, d_{s}=0.2 \mu \mathrm{m}, L_{w}=20 \mu \mathrm{m}$, $\theta=7^{\circ}$, and $R_{f} \leq 1 \%$ were used in this experiment. The gain ripple measured at an injected current of $200 \mathrm{~mA}$ was as small as $0.1 \mathrm{~dB}$. The FWHM of the horizontal and vertical FFPs was almost symmetric $\left(20^{0} \times 20^{0}\right)$. Fiber-to-fiber gains as high as $26 \mathrm{~dB}$ were achieved.

\section{REFERENCES}

[1] H. Hatakeyama, T. Tamanuki, K. Mori, T. Ae, T. Sasaki, and M. Yamaguchi, "Uniform and high-performance eight-channel bent waveguide SOA array for hybrid PICs," IEEE Photon. Technol. Lett., vol. 13, pp. 418-420, May 2001.

[2] H. K. Kim, S. Chandrasekhar, A. Srivastava, C. A. Burrus, and L. Buhl, "10Gbit/s based WDM signal transmission over $500 \mathrm{~km}$ of NZDSF using semiconductor optical amplifier as the in-line amplifier," Electron. Lett., vol. 37, no. 3, pp. 185-187, 2001.

[3] T. Durhuus, B. Mikkelsen, C. Joergensen, S. L. Danielsen, and K. E. Stubkjar, "All-optical wavelength conversion optical amplifiers," $J$. Lightwave Technol., vol. 14, pp. 942-954, June 1996.

[4] T. Saitoh and T. Mukai, "Recent progress in semiconductor laser amplifiers," J. Lightwave Technol., vol. 6, pp. 1656-1664, Nov. 1988.

[5] T. Mukai and Y. Yamamoto, "Gain, frequency bandwidth, and saturation output power of AlGaAs DH laser amplifiers," IEEE J. Quantum Electron., vol. QE-17, no. 6, pp. 1028-1034, 1981.

[6] J. Lee, T. Tanaka, S. Sasaki, S. Uchiyama, M. Tsuchiya, and T. Kamiya, "Novel design procedure of broad-band multiplayer antireflection coatings for optical and optoelectronic devices," J. Lightwave Technol., vol. 16, no. 5, pp. 884-891, 1998 .

[7] W. Rideout, R. Holmstron, J. Lacourse, E. Meland, and W. Powazinik, "Ultra-low-reflectivity semiconductor optical amplifiers without antireflection coatings," Electron. Lett., vol. 26, no. 1, pp. 36-38, 1990.

[8] I. Cha, M. Kitamura, and I. Mito, " $1.5 \mu \mathrm{m}$ band traveling-wave semiconductor optical amplifiers with window facet structure," Electron. Lett., vol. 25, no. 3, pp. 242-243, 1989.
[9] B. Mersali, H. J. Brückner, M. Feüillade, S. Sainson, A. Ougazzaden, and A. Carenco, "Theoretical and experimental studies of a spot-size transformer with integrated waveguide for polarization insensitive optical amplifiers," J. Lightwave Technol., vol. 13, pp. 1865-1872, Aug. 1995.

[10] A. E. Kelly, I. F. Lealman, L. J. Rivers, S. D. Perrin, and M. Silver, "Low noise figure (7.2dB) and high gain ( $29 \mathrm{~dB})$ semiconductor optical amplifier with a single layer AR coating," Electron. Lett., vol. 33, no. 6, pp. 536-538, 1997.

[11] S. Kitamura, H. Hatakeyama, T. Tamanuki, T. Sasaki, K. Komatsu, and M. Yamaguchi, "Angled-facet S-bend semiconductor optical amplifiers for high-gain and large-extinction ratio," IEEE Photon. Technol. Lett., vol. 11, pp. 788-790, 1999.

[12] C. E. Zah, R. Bhat, S. G. Menocal, N. Andredakis, F. Favire, C. Caneau, M. A. Koza, and T. P. Lee, " $1.5 \mu \mathrm{m}$ GaInAsP angled-facet flared-waveguide traveling-wave laser amplifier," IEEE Photonics Technol. Lett., vol. 2, pp. 46-47, Jan. 1990.

[13] J. R. Kim, J. S. Lee, S. Park, M. W. Park, J. S. Yu, S. D. Lee, A. G. Choo, T. I. Kim, and Y. H. Lee, "Spot-size converter integrated polarization insensitive semiconductor optical amplifiers," IEEE Photon. Technol. Lett., vol. 11, pp. 967-969, Aug. 1999.

[14] Z. Wang, B. Mikkelsen, B. Pedersen, K. E. Stubkjaer, and D. S. Olessen, "Coupling between angled-facet amplifiers and tapered lens-ended fibers," J. Lightwave Technol., vol. 9, pp. 49-55, Jan. 1991.

[15] M. J. Robertson, P. C. Kendall, S. Ritchie, P. W. A. Mcilory, and M. J. Adams, "The weighted index method: A new technique for analyzing planar optical waveguides," J. Lightwave Technol., vol. 7, pp. 2105-2111, Dec. 1989

[16] M. Gault, P. A. Mawby, and M. S. Towers, "Solution to the wave equation in the numerical simulation of buried heterostructure," Proc. Inst. Elect. Eng., vol. 140, pp. 44-48, Jan. 1993.

[17] P-.A Besse, J. S. Gu, and H. Melchior, "Reflectivity minimization of semiconductor amplifiers with coated and angled facets considering two-dimensional beam profiles," IEEE J. Quantum Electron., vol. 27, pp. 1830-1836, June 1991

[18] A. Vukovic, P. Sewell, T. Benson, and P. C. Kendall, "Advances in facet design for buried lasers and amplifiers," IEEE J. Select. Topics Quantum Electron., vol. 6, pp. 175-184, Jan. 2000.

[19] D. J. Gallant, M. L. Tilton, D. J. Bossert, J. D. Barrie, and G. C. Dente, "Optimized single-layer antireflection coatings for semiconductor lasers," IEEE Photon. Technol. Lett., vol. 9, pp. 300-302, Mar. 1997.

[20] L. Atternas and L. Thylen, "Single-layer antireflection coating of semiconductor lasers: Polarization properties and the influence of the laser structure," J. Lightwave Technol., vol. 7, pp. 426-430, Feb. 1989.

[21] W. Hellmich and P. P. Deimel, "Optimal AR-coating for optical waveguide devices," J. Lightwave Technol., vol. 10, pp. 469-476, Apr. 1992.

[22] P. Sewell, M. Reed, T. M. Benson, and P. C. Kendall, "Full vector analysis of two-dimensional angled and coated optical waveguide facetes," IEEE J. Quantum Electron., vol. 33, pp. 2311-2318, Dec. 1997.

[23] Z. Wang, B. Mikkelsen, K. F. Stubkjaer, and D. S. Olessen, "Single-layer coating for angled facet amplifier," Electron. Lett., vol. 25, no. 17, pp. 1139-1141, 1989.

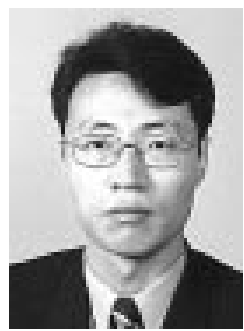

Jongin Shim was born in Kangreung, Korea, in 1960. He received the B.S. and M.S. degrees in electronics from Seoul National University, Seoul, Korea, in 1983 and 1985, respectively, and the Ph.D. degree from the Department of Physical Electronics, Tokyo Institute of Technology, Tokyo, Japan, in 1992. His dissertation was mainly concerned with static and dynamic properties of single-mode lasers such as DR distributed Bragg reflectors and distributed feedback lasers.

From 1985 to 1988 , he was with the Electronics and Telecommunications Research Institute (ETRI), Taejon, Korea, where he worked on InGaAsP-InP optoelectronics lasers. In 1992, he joined the Optoelectronics Research Laboratory of NEC, Tsukuba, Japan, where he conducted research on tunability and coherence of semiconductor lasers and their fabrication technology by selective MOVPE growth. He has since joined the Department of Electrical and Computer Engineering, Hanyang University, Ansan, Korea, as an Associate Professor. His primary interests are optoelectronic semiconductor device modeling and optoelectronic packaging.

Dr. Shim received a Student Award in Excellence in 1992 from the Tokyo Institute of Technology. 
Jongryeol Kim received the B.S. and M.S. degrees in solid-state physics from Seoul National University, Seoul, Korea, in 1987 and 1989, respectively, and the $\mathrm{Ph} . \mathrm{D}$. degree in applied optics from Korea Advanced Institute of Science and Technology, Daejon, Korea, in 2000.

Since 1989, he has been with Samsung Electronics Company, Kyungki-Do, Korea, where he is currently a Principal Engineer of the Fiber-Optics Division. His main research interests are in the development of novel optoelectronic devices for the future optical networks.

Donghoon Jang was born in Kangreung, Korea, in 1960. He received B.S degree from Seoul National University, Seoul, Korea, in 1983, and the Ph.D. degree in materials science from Korea Advanced Institute of Science and Technology (KAIST), Daejon, Korea, in 1988.

He was a Senior Engineer at Electronics and Telecommunication Research Institute (ETRI), Daejon, Korea from 1988 to 2000, where he was responsible for the development of GaInAsP-InP optoelectronics devices. He was a Visiting Scientist at Sophia University, Japan, in 1991, where he worked on visible semiconductor lasers using GSMBE. In 2000, he joined Samsung Electronics Optoelectronics Division, Suwon, Korea, as Manager of the R\&D Group. His research interests are epitaxial growth, fabrication of semiconductor lasers, characteristics of modules for transmission, high-speed optoelectronic semiconductor devices, and integrated modules for optical fiber communications.

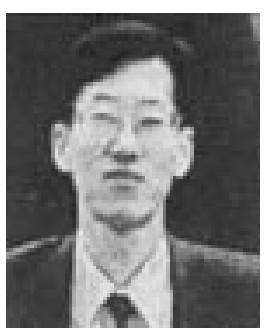

Yungseon Eo received the B.S. and M.S. degrees in electronic engineering from Hanyang University, Seoul, Korea, in 1983 and 1985, respectively, and the Ph.D. degree in electrical engineering from the University of Florida, Gainesville, in 1993.

From 1986 to 1988 , he was with the Korea Telecommunication Authority Research Center, Seoul, Korea, where he performed telecommunication network planning and software design. From 1993 to 1994, he performed $s$-parameter-based BJT device characterization and modeling for high-speed circuit design at Applied Micro Circuits Corporation, San Diego, CA. From 1994 to 1995, he was with the Research and Development Center of LSI Logic Corporation, Santa Clara, CA, where he worked in the area of signal integrity characterization and modeling of high-speed CMOS circuits and interconnects. In 1995, he joined the Department of Electrical and Computer Engineering, Hanyang University, Ansan, Korea, where he is currently an Associate Professor. His research interests are high-frequency characterization and modeling of integrated circuits and interconnects, and high-speed VLSI circuit packaging.

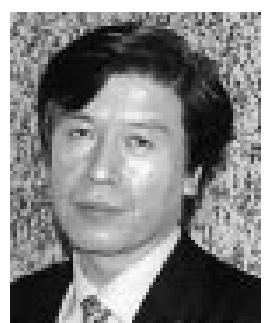

Shigehisa Arai (M'83) was born in Kanagawa, Japan, in 1953. He received the B.E., M.E., and D.E. degrees in electronics from Tokyo Institute of Technology, Tokyo, Japan, in 1977, 1979, and 1982, respectively.

During his doctoral work, he developed the two-phase solution LPE growth of GaInAsP-InP in the emission wavelength of $1.1-1.6 \mu \mathrm{m}$ and $\mathrm{BH}$ lasers in the $1.5-1.6-\mu \mathrm{m}$ region, as well as dynamic single-mode lasers. In 1982, he joined the Department of Physical Electronics, Tokyo Institute of Technology, as a Research Associate. From 1983 to 1984, he was with AT\&T Bell Laboratories, Holmdel, NJ. He became an Associate Professor with the Department of Physical Electronics in 1987, and a Professor with the Research Center for Quantum Effect Electronics in 1994, both at Tokyo Institute of Technology. He has been engaged in the research on photonic integrated devices, such as dynamic-single-mode/wavelength tunable semiconductor lasers, quantum-wire/quantum-box lasers, semiconductor optical amplifiers, and optical switches/modulators. Recently, he also has been engaged in research on low-damage and cost-effective processing technologies for high-performance lasers and photonic integrated circuits.

Dr. Arai received an Excellent Paper Award from the Institute of Electronics, Information and Communication Engineers (IEICE) of Japan in 1998, and the Michael Lunn Memorial Award from IPRM (Indium Phosphide and Related Materials) in 2000. He is a member of the IEEE LEOS, the Optical Society of America (OSA), IEICE, and Japan Society of Applied Physics. 